\title{
An 83-year-old female with worsening dyspnoea and bleeding from a cavernostomy
}

\author{
M. Scharitzer*, G. Dekan\#, L. Stiebellehner" and A.A. Bankier*
}

\section{CASE HISTORY}

An 83-yr-old female presented with a history of worsening dyspnoea. In 1952, the patient had undergone surgical treatment for progressive pulmonary tuberculosis (TB) of the left-upper lobe. Treatment had consisted of an artificial pneumothorax and a cavernostomy via incision and drainage of a tuberculous cavity. Since surgery, the patient had cared for the cavernostomy herself by occasionally draining secretions using a plastic tube prescribed by a general practitioner. There had been no other post-surgical surveillance over almost 50 yrs. The patient had no history of smoking.

On admission, the patient was in reduced general condition and reported recurrent local bleedings from the cavernostomy. Physical examination revealed that the cavernostomy was occluded by a polypoid mass, presumably located within the cavity. Both the mass and the cavernostomy were bleeding slightly. There was no palpable local lymphadenopathy. Auscultation was unremarkable. The serum level of Creactive protein was markedly raised at $81 \mathrm{mg} \cdot \mathrm{L}^{-1}$ (normal: $<10 \mathrm{mg} \cdot \mathrm{L}^{-1}$ ). The blood serum levels of alkaline phosphatase (228 U. $\mathrm{L}^{-1}$; normal: $\left.<170 \mathrm{U} \cdot \mathrm{L}^{-1}\right)$ and $\gamma$-glutamyltransferase $(23$ $\mathrm{U} \cdot \mathrm{L}^{-1}$; normal: $4-18 \mathrm{U} \cdot \mathrm{L}^{-1}$ ) were slightly elevated, as was the blood serum level of uric acid $\left(81 \mathrm{mg} \cdot \mathrm{L}^{-1}\right.$; normal: 25 $\left.60 \mathrm{mg} \cdot \mathrm{L}^{-1}\right)$. All other laboratory tests, including haematocrit, were within the normal ranges.

The chest radiograph obtained on admission is shown in figure 1. Contrast-enhanced computed tomography (CT) performed on the same day, before and after drainage of fluid from the cavity, is shown in figures 2 and 3, respectively.
Figure 4 shows a CT section taken while the cavernostomy was drained by the patient using the plastic tube, as described above.

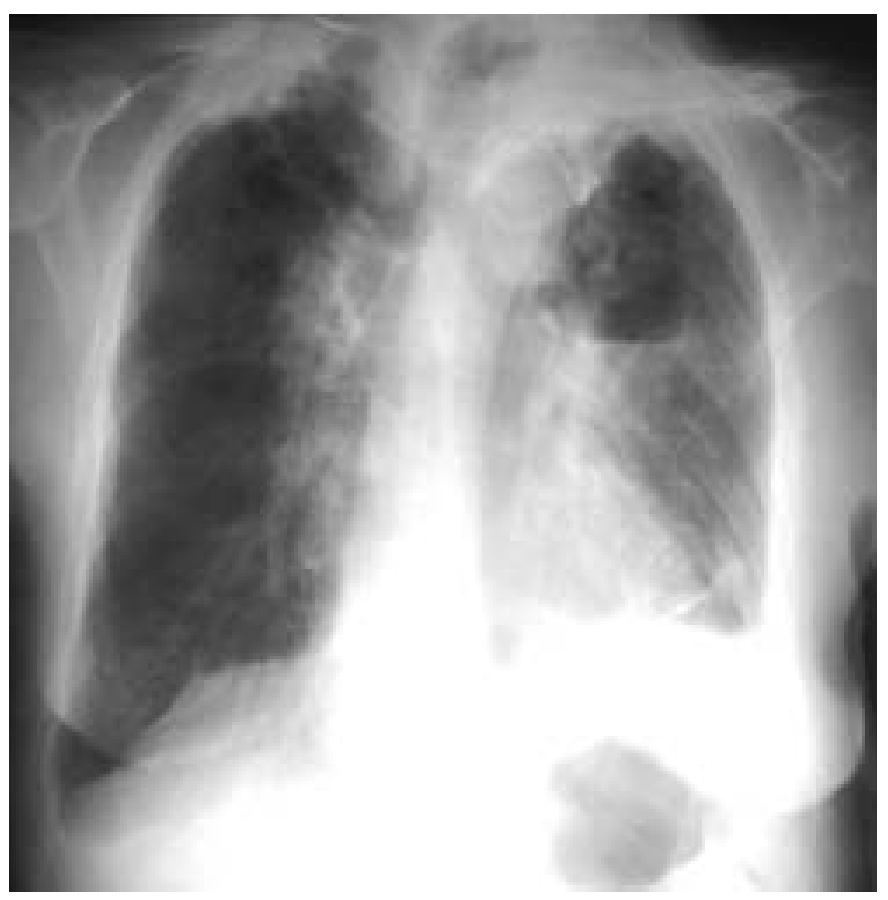

FIGURE 1. Postero-anterior chest radiograph obtained at admission. 
Repeated percutaneous biopsies harvested multiple tissue specimens of the intracavitary mass. Samples from these specimens are shown in figure 5.
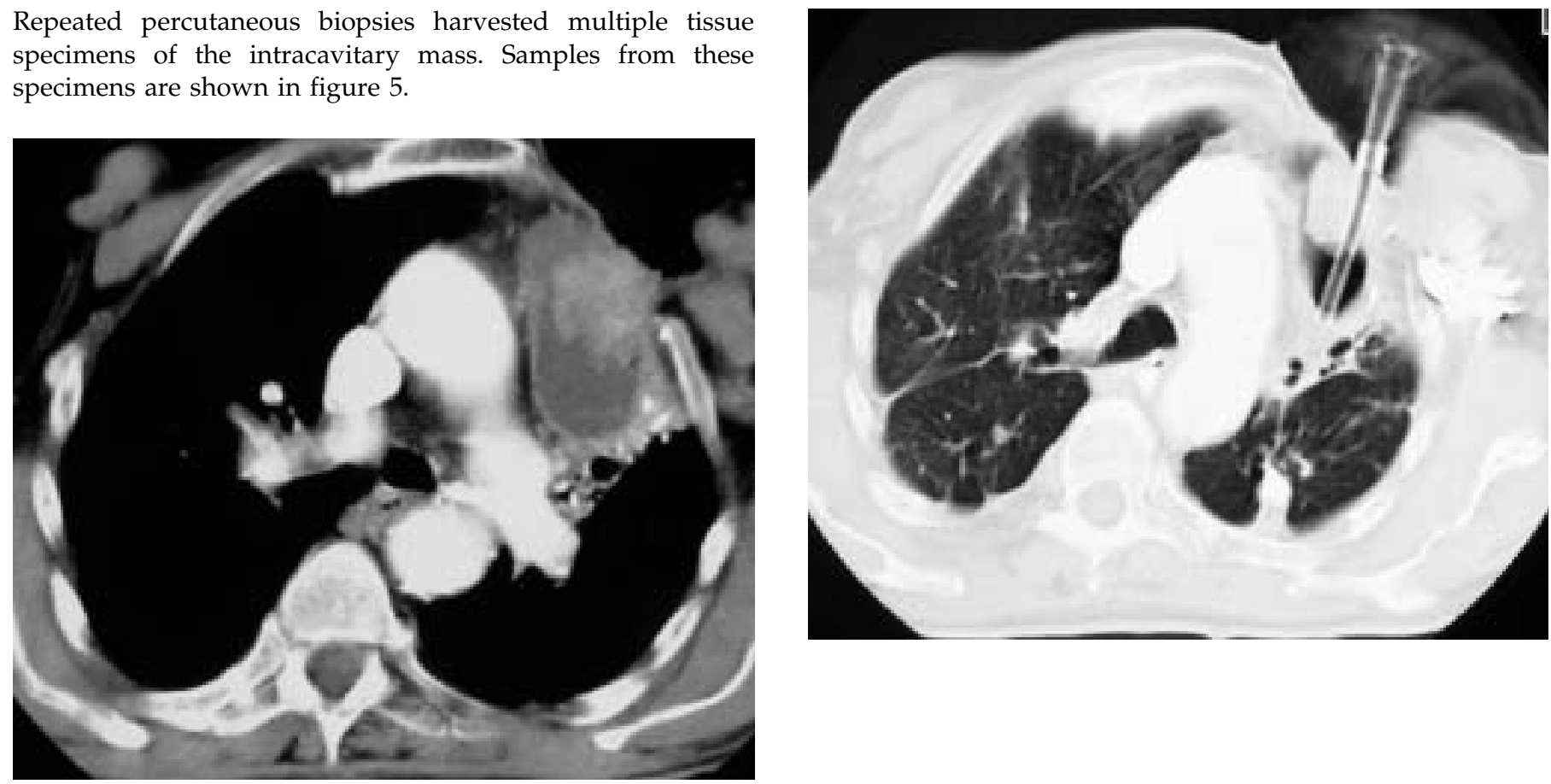

FIGURE 2. Contrast-enhanced computed tomography sections at the level of the pulmonary arteries before drainage of fluid from the cavernostomy.

FIGURE 4. Contrast-enhanced computed tomography scan at the level of the carina showing the drainage of the cavernostomy by the patient using a plastic tube, as described previously.
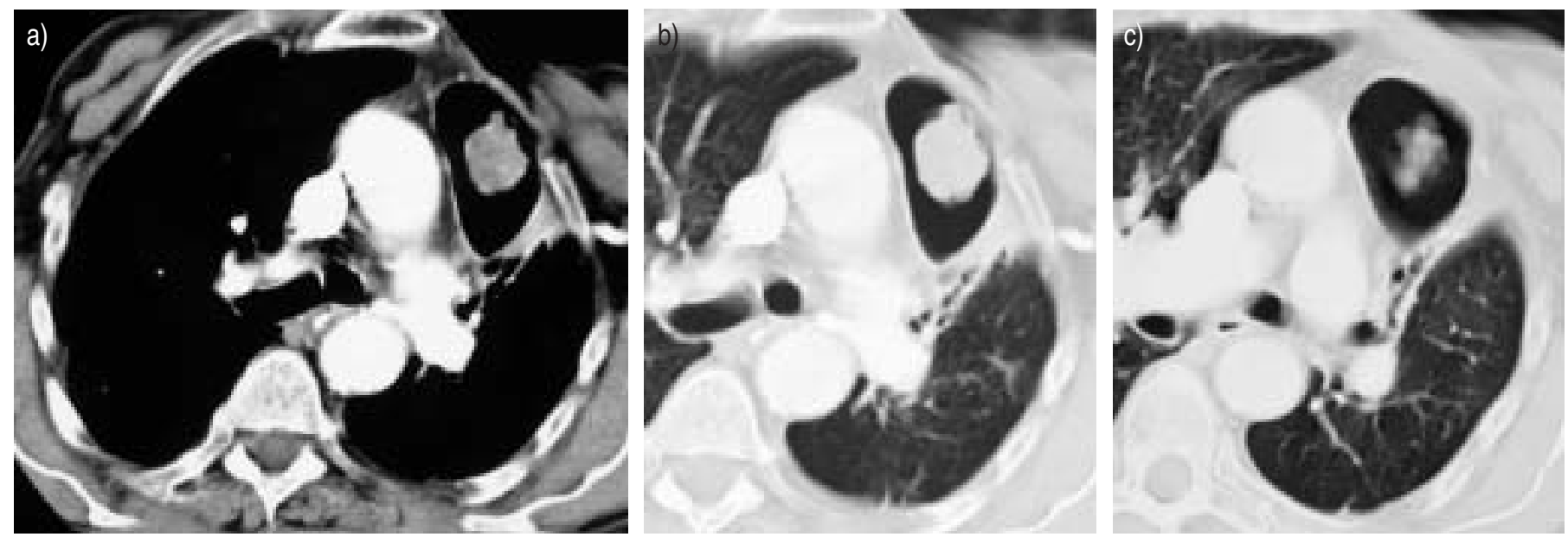

FIGURE 3. Computed tomography sections at two different anatomical levels after drainage of fluid from the cavity with a) soft tissue and b, c) lung window settings. 

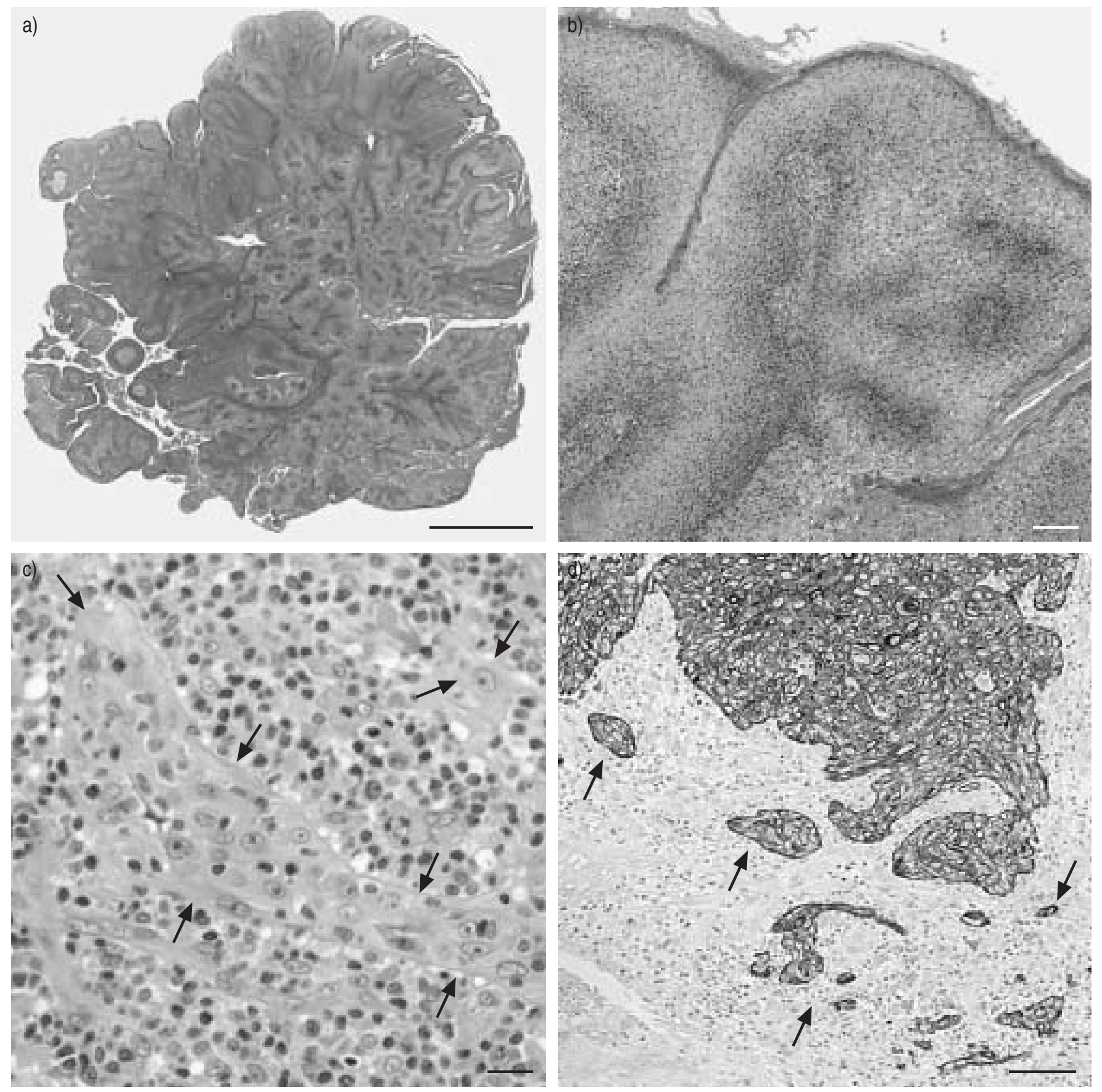

FIGURE 5. Lung surgical biopsies stained with $a, b, c)$ haematoxylin and eosin and d) following immunohistochemistry with Lu-5 (a pan anti-cytokeratin antibody). Scale bars: a) $=5 \mathrm{~mm} ; \mathrm{b})=2 \mathrm{~mm} ; \mathrm{c})=500 \mu \mathrm{m}$; and d) $=1 \mathrm{~mm}$.

BEFORE TURNING THE PAGE, INTERPRET THE CHEST RADIOGRAPH, COMPUTED TOMOGRAPHY SCANS AND HISTOLOGY SLIDES, AND SUGGEST A DIAGNOSIS. 


\section{INTERPRETATION}

\section{Chest radiographs and $\mathrm{CT}$ scans}

Chest radiography confirmed both the presence of a cavity in the left-upper lobe and the presence of an intracavitary mass $\sim 3 \mathrm{~cm}$ in diameter (fig. 1). The chest radiograph also showed bilateral pleural thickening at the level of the lung apices and a reduction in volume of the left hemithorax. The lung parenchyma displayed bilaterally scattered and partly calcified consolidations suggestive of fibrosis (fig. 1).

CT revealed that the intracavitary mass was solid, multilobulated, had soft tissue attenuation and showed inhomogeneous contrast enhancement (fig. 2). The mass had broad contact with the anterior area of the wall of the cavity (fig. 3). The wall of the cavity itself had broad contact with the anterior area of the chest wall (fig. 3). The presence of apical pleural thickening (fig. 4) and of partly calcified parenchymal consolidations suggestive of parenchymal fibrosis was confirmed. The only mediastinal abnormality was a solitary, slightly enlarged ipsilateral lymph node.

\section{Pathology}

Material submitted for histological work-up was stained with haematoxylin and eosin. It consisted of several nodular, greyish and moderately firm fragments, measuring $4 \times 2 \mathrm{~cm}$ overall. Microscopically, loose fibrovascular cores with stratified, moderately dysplastic, squamous epithelium with focal changes mimicking koilocytes were predominant. The largest fragment is shown in figures $5 \mathrm{a}$ and $\mathrm{b}$. Fragments smaller in size were shown to be embedded in the sparsely inflamed stromal areas with small invasive discohesive groups of moderately dysplastic cells (fig. $5 \mathrm{c}$ and d). Following MIB-1 antibody staining, focal areas of dysplastic epithelium showed $>70 \%$ nuclear reactivity. In situ hybridisation for human papilloma virus (HPV) types 6/11, 16/18 and 31/33/35 and hybrid capture hc2 HPV DNA test were negative.

\section{Diagnosis: Highly differentiated invasive squamous cell carcinoma.}

\section{CLINICAL COURSE}

Due to both the reduced general condition of the patient and the patient's personal reluctance, surgical excision of the mass and the cavity was not performed. However, PCR analysis for mycobacteria of the punctate and the hybrid capture HPV test for HPVs were performed, but both tests were negative. On her own request, the patient left hospital under antibiotic therapy. She failed to present at the scheduled clinical control examinations and was finally lost to follow-up.

\section{DISCUSSION}

The present case illustrates the clinical, radiological and pathological sequelae of a surgical treatment for TB that dates from the pre-antibiotic era. Artificially induced collapse of the lung as a potential cure for TB was episodically mentioned in the 1600s. Systematically performed therapeutic pneumothorax procedures to treat TB were first reported in 1888 and introduced by Forlanini [1, 2]. Both surgical resection of isolated diseased parts of the lung and thoracoplasty remained common treatments up to the 1950s [1, 3]. After effective chemotherapeutic agents had been introduced, surgical treatments for TB, notably the use of therapeutic pneumothorax, were rapidly discontinued. In Central Europe, this technique might be considered as "historical". However, in Eastern Europe surgery has kept an important place in the treatment of TB. Moreover, in North America it has now become highly fashionable for multidrug-resistant TB [4].

The present patient showed a late potential complication of this technique. The complication consisted of a solid mass that developed within the post-surgical cavity. The clinical suspicion of malignancy was supported by the patient's reduced general condition and the recurrent bleedings from the cavernostomy. Moreover, there was the theoretical possibility of seeding of tumour cells due to the patient's own cannulation of the cavernostomy. However, based on the CT findings alone, no definite distinction could be made between a benign reactive lesion, a superimposed lung cancer or a scar carcinoma. The differential diagnosis was nevertheless weighted toward malignancy because benign masses, such as apical lesions, are found only in a small minority of patients with a history of TB [5]. Finally, bleeding of the tumour, as seen in this patient, is a strong indicator of malignancy, as it is in exophytic bronchial carcinoma, colon carcinoma and malignant melanomas.

The potential pathogenesis of squamous cell carcinoma (scar cancer) in $\mathrm{TB}$, and the issue as to whether the scar precedes or follows the development of the carcinoma have been a controversial subject [6-11]. In the patient, there was no history of exposure to known carcinogens that could have been associated with the development of carcinoma. Moreover, the lesion developed in an area of parenchymal fibrosis which was damaged after previous parenchymal TB. Finally, the lesion in the patient was located adjacent to the anatomical region where surgery was performed, thereby suggesting a potential pathogenetic relationship.

Other late post-operative complications of therapeutic pneumothorax are related to infections promoted by the development of fluid within the cavernostomy, and the formation of bronchopleural or extrapleural fistulae or pyogenic empyema [12]. The presence of a fungus ball in the patient was unlikely to be due to the broad contact of the solid mass with the anterior area of the cavity, and to the resulting absence of mobility and gravity dependence. Furthermore, laboratory tests were not congruent with infection.

To confirm malignancy, repeated percutaneous biopsies were requested for this patient. Indeed, the literature reports that the definite differentiation between the benign or the malignant nature of this complication can be difficult, and even impossible, to establish, despite multiple biopsies [13]. In about one-third of cases, solitary papillomata are associated with dysplasia and carcinoma in situ or undergo malignant transformation [14]. Electron microscopy of the epithelium reveals atypical cells invading adjacent tissue or lymphatics [14]. In the present case, careful histopathological analysis of serial sections of the lesion revealed distinct foci of moderately dysplastic cells embedded in the sparsely inflamed stroma, consistent with microscopic signs of invasiveness, and overall with malignancy [13]. Nevertheless, the earliest stages of malignant transformation, including loss of normal cell structure and stromal changes, remain very difficult to 
differentiate from reactive squamous epithelium with cytological atypia [14]. Furthermore, infections with HPV may contribute to the occurrence of severe dysplasia in squamous papilloma [13]. However, in this patient, virus typing by in situ hybridisation and hybrid capture assay did not confirm the presence of HPV.

In summary, the present case documents a late and rare complication of a historical surgical treatment for tuberculosis. The clinical, radiological and pathological features of this complication are detailed. It also emphasises the difficulties in establishing the correct diagnosis, despite a thorough radiological and histological work-up.

\section{REFERENCES}

1 Rubin SA. Tuberculosis. Captain of all these men of death. Rad Clin North Am 1995; 33: 619-639.

2 Chan ED, Laurel V, Strand MJ, et al. Treatment and outcome analysis of 205 patients with multidrug-resistant tuberculosis. Am J Respir Crit Care Med 2004; 169: 1103-1109.

3 Sakula A. Carlo Forlanini, inventor of artificial pneumothorax for treatment of pulmonary tuberculosis. Thorax 1983; 38: 326-332.

4 Monaldi V. Endocavitary aspiration in the treatment of lung abscess. Dis Chest 1956; 29: 193-201.
5 Woodring JH, Vandiviere HMJ, Fried AM, et al. Update: the radiographic features of pulmonary tuberculosis. AJR Am J Roentgenol 1986; 146: 497-506.

6 Richardson S, Hirsch A, Ruffie P, et al. Relationship between tuberculous scar and carcinomas of the lung. Eur J Radiol 1987; 7: 163-164.

7 Auerbach O, Garfinkel L, Parks VR. Scar cancer of the lung: increase over a 21 year period. Cancer 1979; 43: 636-642.

8 Bakris GL, Mulopulos GP, Korchik R, et al. Pulmonary scar carcinoma. A clinicopathologic analysis. Cancer 1983; 52: 493-497.

9 Kung IT, Lui IO, Loke SL, et al. Pulmonary scar cancer. A pathologic reappraisal. Am J Surg Pathol 1985; 9: 391-400.

10 Cagle PT, Cohle SD, Greenberg SD. Natural history of pulmonary scar cancers. Clinical and pathologic implications. Cancer 1985; 56: 2031-2035.

11 Dacosta NA, Kinare SG. Association of lung carcinoma and tuberculosis. J Postgrad Med 1991; 37: 185-189.

12 Weissberg D, Weissberg D. Late complications of collapse therapy for pulmonary tuberculosis. Chest 2001;120: 847-851.

13 Travis WD, Brambilla E, Müller-Hermelink HK, Harris CC, eds. Pathology and Genetics of Tumours of the Lung, Pleura, Thymus and Heart. Lyon, IARC Press, 2004.

14 Colby TV, Koss MN, Travis WD. Atlas of tumor pathology, series III, fascicle 13; tumors of the lower respiratory tract American Registry of Pathology 1995: 51-53. 